\title{
Article \\ Combining Ability and Heterosis of Algerian Saharan Maize Populations (Zea mays L.) for Tolerance to No-Nitrogen Fertilization and Drought
}

\author{
Meriem Riache ${ }^{1}$, Pedro Revilla ${ }^{2}$ D, Oula Maafi ${ }^{1}$, Rosa Ana Malvar ${ }^{2}{ }^{(D}$ and Abderahmane Djemel ${ }^{1, *}$ \\ 1 Ecole Nationale Supérieure Agronomique, Avenue Hassan Badi, El Harrach, Algiers 16051, Algeria; \\ m.riache93@gmail.com (M.R.); oulamaafi@gmail.com (O.M.) \\ 2 Misión Biológica de Galicia (CSIC), Apartado 28, 36080 Pontevedra, Spain; previlla@mbg.csic.es (P.R.); \\ rmalvar@mbg.csic.es (R.A.M.) \\ * Correspondence: djemeldahmane@yahoo.fr; Tel.: +213-558-410418
}

check for updates

Citation: Riache, M.; Revilla, P.; Maafi, O.; Malvar, R.A.; Djemel, A. Combining Ability and Heterosis of Algerian Saharan Maize Populations (Zea mays L.) for Tolerance to

No-Nitrogen Fertilization and Drought. Agronomy 2021, 11, 492. https://doi.org/10.3390/ agronomy11030492

Received: 16 February 2021

Accepted: 3 March 2021

Published: 6 March 2021

Publisher's Note: MDPI stays neutral with regard to jurisdictional claims in published maps and institutional affiliations.

Copyright: (c) 2021 by the authors. Licensee MDPI, Basel, Switzerland. This article is an open access article distributed under the terms and conditions of the Creative Commons Attribution (CC BY) license (https:// creativecommons.org/licenses/by/ $4.0 /)$.

\begin{abstract}
Drought and low nitrogen are major stresses for maize (Zea mays L.), and maize populations from the Sahara Desert are potential sources of stress tolerance. The objectives were to assess the tolerance and varietal and heterosis effects of Algerian populations under no-nitrogen fertilization and water stress. A diallel among six Algerian maize population was evaluated under drought (300 mm irrigation) vs. control $(600 \mathrm{~mm})$ and no-nitrogen fertilization vs. $120 \mathrm{kh} \mathrm{ha}^{-1} \mathrm{~N}$ fertilization. Genotypes showed significant differences and genetic effects for water- and nitrogen-stress tolerance. We propose a reciprocal recurrent selection to take advantage of additive and non-additive effects, using AOR and IGS, since they showed good performance in optimum and stress conditions, for improving yield heterosis for AOR $\times$ IGS. Negative effects are not expected on plant height, anthesissilking interval or early vigor. These populations and BAH could be sources of inbred lines tolerant to drought and no-nitrogen fertilization. There was no relationship between origin and genetic group and stress tolerance per se or as parents of tolerant crosses. These populations and crosses could be used as base material among Algerian populations, for breeding programs focusing on tolerance to water or nitrogen stress.
\end{abstract}

Keywords: maize; Algerian germplasm; varietal effect; heterosis; drought; nitrogen fertilization

\section{Introduction}

Maize (Zea mays L.) is one of the most important crops due to its large demand for both human and animal food and wider adaptability [1,2]. Maize is cultivated across a wide range of climatic conditions from tropical to temperate areas [3]. The food demand is predicted to double by 2050 [4]; however, the food production systems are greatly threatened by water availability and soil degradation, and these challenges are expected to increase with climatic change $[5,6]$. Therefore, abiotic stresses are the major threat to agriculture production worldwide [7], and a nearly 20-million-hectare area is estimated to be water-scarce by 2025 [8]. Furthermore, models of climate change predict that drought stress will become the primary agricultural limitation $[9,10]$.

In developing countries, climate change will cause severe yield declines, especially in Africa, where food security is already threatened [11]. Drought stress is one of several reasons for the differences between mean production levels of temperate versus tropical regions [12]. In addition, low soil-nitrogen availability is responsible for significant yield losses in maize [13-15]. Breeding for low-nitrogen tolerance is the most effective and sustainable approach to mitigate this problem in developing countries $[14,16]$. Nitrogen and water, separately or in combination, are two of the most critical factors in maize production worldwide $[11,17,18]$. In order to establish a successful breeding program for improving low nitrogen and/or drought tolerance, available maize germplasm should be screened for 
agronomic and physiological performance under stress conditions to identify tolerant genotypes that could be used directly or indirectly as suitable sources for developing tolerant hybrids [15]. Previous reports suggest that maize varieties selected for tolerance to drought may possess tolerance to low nitrogen conditions since the physiological mechanisms for tolerance may be similar in both [19].

Genetic diversity is crucial for plant breeding [20,21], and maize has larger genetic diversity than any other domesticated grass [22-24]. However, only $5 \%$ to $10 \%$ of that diversity is used in maize breeding programs [25]. This limitation causes serious problems in sustaining yield production, especially in unfavorable areas in developing countries [26]. Therefore, using other sources of alleles is of paramount importance and, according to Al-Naggar et al. [15], a maize breeding program against all stresses requires large genetic diversity. In general, maize landraces or traditional varieties are considered as the main donors of resistance genes [27-29], allowing plant breeders to select improved varieties and inbred lines by enhancing traits controlling agricultural productivity and performance [30].

Maize landraces can be used to increase the narrow genetic base of the existing inbred lines and varieties [31]. The International Maize and Wheat Improvement Center (CIMMYT) collected and conserved various maize accessions from various countries and many studies on Sub-Saharan African maize were carried out. However, little works are nowadays available about maize genetic diversity in North Africa, except a few reports about Algerian maize populations from the Sahara Desert [32,33]. Maize from the Sahara Desert could constitutes a potential source of stress tolerance because of its adaptation to biotic or abiotic stress. Accordingly, Hawtin et al. [27] stated that favorable alleles against abiotic stress may be found in extreme environments. Beyene et al. [34] also reported that the maize variability available in African countries is large and could be used in maize breeding programs. In 2009, some maize populations were collected from a subtropical area in the Algerian Sahara. Phenotypic [32,35-37] and genetic [33,38,39] studies revealed that Algerian maize exhibited high diversity and could provide new alleles for drought conditions. Cherchali et al. [40] studied the heterotic patterns among Algerian germplasm. Those previous reports showed a high degree of genetic divergence among Algerian maize populations and suggested that Algerian maize could be incorporated into breeding programs as donor of favorable alleles for improving early growth (AOR and IGS), reducing flowering time (AOR, MST, and BAH), and increasing yield production (IGS), especially under drought conditions.

Our objectives were (i) to estimate varietal and heterosis effect of Algerian Saharan populations and their crosses for grain yield and other agronomic traits under no-nitrogen fertilization and drought conditions, and (ii) to select the most promising populations and crosses for breeding for stress tolerance.

\section{Materials and Methods}

\subsection{Plant Materials}

In 2009, maize germplasm was collected in oases of the Algerian Sahara [32]. Based on genetic distances and geographic origin previously defined by Aci et al. [33], six Algerian populations representing that collection (Supplementary Table S1) were crossed in a diallel mating design without reciprocals in 2013 to produce 15 hybrids [40]. These populations belong to three genetic pools: Cluster I (IGS: from the most southern area (province of Tamanrasset); Cluster II (accessions coming from the center of the prospected area, Adrar (AOR, IZM, and MST), with the SHH population from the north of Algeria as a small group; and Cluster III from Bechar province (BAH). In addition, the distance between their collection sites was $>50 \mathrm{~km}$ [40]. The diallel crossing system was made as described by Cherchali et al. [40]. Briefly, for each pair of populations, 60 pairs of plants were used to produce 60 crosses, and all kernels produced were bulked for each hybrid. 


\subsection{Field Trials}

The fifteen crosses and their parents, plus four checks (the hybrid EP17 $\times$ EP42 from Spain; the two synthetic varieties, EPS20 (originated from eight Reid inbred lines) and EPS21 (originated from eight non-Reid inbred lines); and their respective cross, EPS20 $\times$ EPS21, were evaluated under both drought and no-nitrogen fertilization, in Algiers, Algeria $\left(36^{\circ} 43^{\prime} 16^{\prime \prime} \mathrm{N}, 3^{\circ} 09^{\prime} 03^{\prime \prime} \mathrm{E}, 36 \mathrm{~m}\right.$ altitude, with $600 \mathrm{~mm}$ of annual rainfall). Trials were sown during the growing seasons of 2018 (3 May) and 2019 (18 May). The driest months were April, May, June, and July (Figure 1).

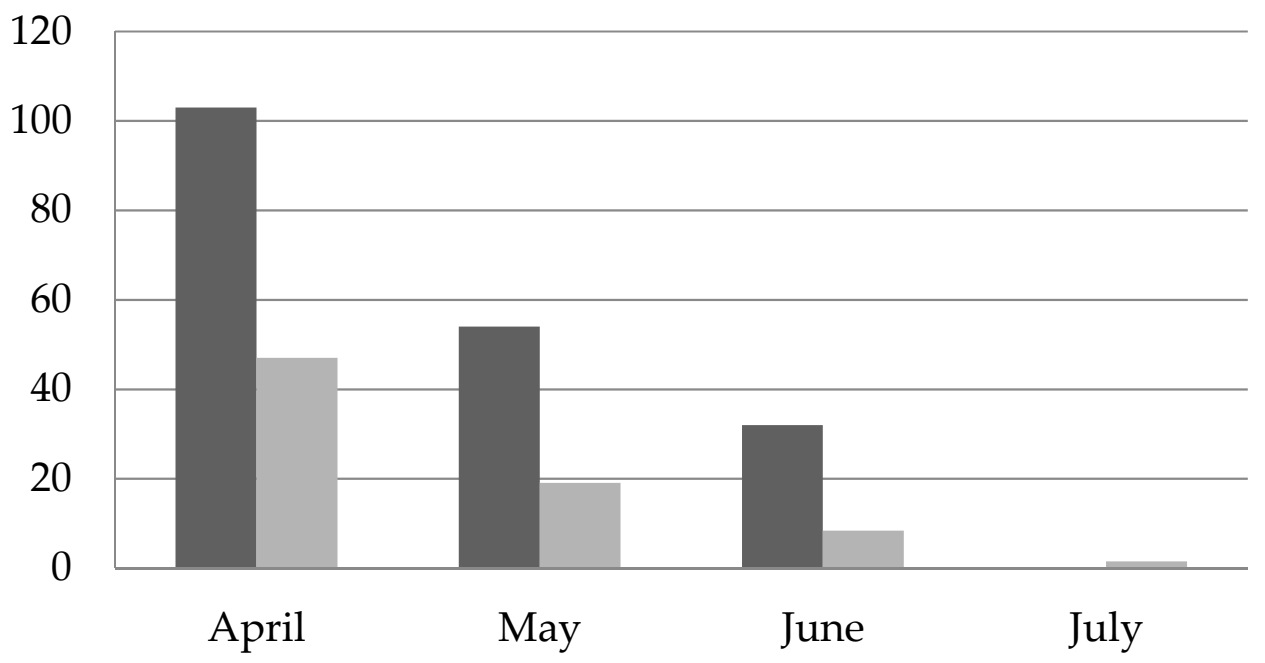

Figure 1. Rainfall (mm) during maize growth in Alger in 2018 (dark gray) and 2019 (light gray).

The experimental design was a split-split plot with three repetitions. Each repetition was divided into two main plots that contain the two water regimes (well-watered and water-stressed). The nitrogen treatment ( $0 \mathrm{~kg}$ of nitrogen and $120 \mathrm{~kg}$ of nitrogen) was arranged in the subplots, and the genotypes in the sub-subplots. Each experimental unit was a single-row plot, $6 \mathrm{~m}$ long with $0.7 \mathrm{~m}$ row spacing, and the hills were spaced $0.2 \mathrm{~m}$, to reach a final plant density of approximately 70,000 plants/ha.

Maize under control condition received $600 \mathrm{~mm}$ of water from sowing to postflowering while only $300 \mathrm{~mm}$ was applied under drought conditions. The trials were irrigated by a drip-irrigation system every week, and when the trials received water from rainfall, the exact amount was removed. Soil characterization analyses of the experimental plot were made before planting. Five soil samples, following a diagonal at $30 \mathrm{~cm}$ depth, were collected. The analyses revealed that the soil had a silty texture and present total nitrogen of $110 \mathrm{ppm}$, moderately poor in available nitrogen (20 ppm in both years), and low organic matter content $(1.53 \%)$. Based on the soil test results, the nitrogen fertilizer was applied as Urea (46\%) in two splits: $40 \mathrm{~kg} / \mathrm{ha}(1 / 3)$ at three-leaf growth stage and $80 \mathrm{~kg} / \mathrm{ha}$ $(2 / 3)$ at six-leaves growth stage except in the subplots 0 nitrogen treatment. Plots were planted and harvested by hand. In both years, the following data were recorded: early vigor was scored 5 weeks after sowing, using a 1 to 9 scale $(1=$ weak plant to $9=$ strong plant); anthesis-silking interval (ASI) was computed as the difference between anthesis (days from planting to $50 \%$ of plants shedding pollen) and silking (days from planting to $50 \%$ of plants showing silks); plant height was the distance $(\mathrm{cm})$ from the ground to the first tassel branch of ten plants; and, finally, the grain yield in $\mathrm{kg} / \mathrm{ha}$ (grain weight per hectare adjusted to $14 \%$ moisture).

\subsection{Statistical Analyses}

Combined analyses of variance over years and conditions and individual analysis by each condition over years were made by using the PROC MIXED procedure of SAS 9.4 software [41] to assess the performance of the maize genotypes under drought and no- 
nitrogen fertilization. Years and repetitions were considered as random factors, whilst the genotypes (populations per se and crosses) and treatments were considered as fixed factors. Means among treatments were compared by using Fisher's protected Least Significant Difference (LSD) at $p=0.05$ (Fisher's test). Leaving out the checks, for each combination water $\times$ nitrogen treatment, varietal effects and heterosis effects (average heterosis, varietal heterosis, and specific heterosis) were estimated in the diallel crosses, by following method II of Gardner and Eberhart [42]. The results were calculated by the following linear model:

$$
Y i j=E+\mathrm{b}(e)+\mu \mathrm{v}+1 / 2(v i+v j)+\frac{1}{2}(\mathrm{ev} i+\mathrm{ev} j)+k(h i j+\mathrm{eh} i j)+\text { Error }
$$

where $Y_{i j}$ is the average value obtained for each variety $(i=j)$ or for a cross $(i \neq j)$; $E$ is the year effect; $\mu \mathrm{v}$ is the mean of $\mathrm{n}$ parental genotypes; $\mathrm{b}(e)$ is the effect of repetition within year; $v i$ and $v j$ are the varietal effects for $i$ and $j$, respectively; $k=0$ when $i=j$ and $k=1$ when $i \neq j$; $h i j$ is the overall heterosis effect; evi is the interaction effect of year and population, $i$; and ehij is the interaction of year and heterosis of populations $i$ and $j$. Error is the experimental error. Varietal effect was calculated as the difference between the mean performance of each parent and the mean of all parents, whereas heterosis effect was calculated as the difference between the mean of two parental populations and their cross. In addition, hij is the deviation from mean heterosis observed in the cross of populations $i$ and $j$. And,

$$
h i j=h+h i+h j+s i j
$$

where $h$ is the average heterosis of all crosses, calculated as the difference between the mean of all crosses and the mean of all parents; $h i$ and $h j$ are the parental heterosis contributed by the variety $i$ and $j$ in their crosses, measured as a deviation from the average heterosis; and sij is the specific combining ability effect of the cross between ith and jth parents. All data were analyzed with the DIALLEL-SAS05 program of Zhang et al. [43].

\section{Results}

\subsection{Analyses of Variance and Comparisons of Means}

Combined analysis of variance revealed non-significant differences between years, and most interactions between year and other factors were not significant (Supplementary Table S2). The effects of genotypes, irrigation, and nitrogen treatments were more important than year and most interactions involving years. Differences among genotypes, irrigation, and nitrogen factors were significant for all traits, except plant height among nitrogen treatments. The interactions were significant for irrigation $\times$ nitrogen for plant height and yield.

Under well-watered conditions, differences among genotypes were significant for all traits under both nitrogen treatments. Furthermore, year $\times$ genotypes interaction was generally not significant. Under water-stress conditions, differences among genotypes were significant for all traits with both nitrogen levels (Supplementary Table S3).

As expected, all traits means-except ASI-were reduced under water-stress conditions, and this negative effect was increased by no-nitrogen supply (Table 1 and Supplementary Table S4). The highest average reduction caused by stress was found in yield. Under water stress conditions, early vigor ranged from 4.17 to 5.00 for populations per se and from 4.33 to 5.67 for their crosses with no-nitrogen supply. The crosses involving $\mathrm{IZM}[\mathrm{IZM} \times \mathrm{IGS}$ and IZM $\times \mathrm{SHH}$, (5.67)] were the most vigorous ones. Under optimum conditions, IGS $\times$ BAH $(251.36 \mathrm{~cm})$ had the tallest plants (Supplementary Table S4). For water stress, mean ASI varied from 6.59 days to 4.99 days under no-nitrogen supply and $120 \mathrm{~kg} /$ ha nitrogen supply respectively, and from 2.83 days (with no-nitrogen supply) to 1.59 days (with nitrogen supply) under well-watered conditions. MST and MST $\times$ BAH exhibited short ASI (3.5 days) under water stress conditions with no-nitrogen (Table 1). 
Table 1. Means ${ }^{a}$ of anthesis-silking interval (ASI) and grain yield from a diallel systems with six Algerian maize populations evaluated along with four checks in two years in Algiers, under both managed drought and nitrogen deficiency.

\begin{tabular}{|c|c|c|c|c|}
\hline \multicolumn{5}{|c|}{ ASI $^{b}$ (Days) } \\
\hline & \multicolumn{2}{|c|}{ Water Stress } & \multicolumn{2}{|c|}{ Well-Watered } \\
\hline & Without $\mathbf{N}^{\mathrm{c}}$ & With $\mathbf{N}$ & Without N & With N \\
\hline \multicolumn{5}{|c|}{ Populations } \\
\hline AOR & 7.17 efgh & 3.83 efgh & $2.83 \mathrm{bc}$ & $1.83 \mathrm{bcde}$ \\
\hline BAH & 6.00 fghij & $8.50 \mathrm{bcd}$ & $2.67 \mathrm{bc}$ & $2.33 \mathrm{bcd}$ \\
\hline IGS & 9.83 cde & 5.83 defg & $2.83 \mathrm{bc}$ & 1.33 cde \\
\hline $\mathrm{IZM}$ & $10.75 \mathrm{~cd}$ & $9.5 \mathrm{ab}$ & $3.67 \mathrm{bc}$ & $2.33 \mathrm{bcd}$ \\
\hline MST & $3.50 \mathrm{j}$ & $4.20 \mathrm{efgh}$ & $1.67 \mathrm{c}$ & $0.67 \mathrm{de}$ \\
\hline $\mathrm{SHH}$ & 4.67 ghij & $3.33 \mathrm{fgh}$ & $1.33 c$ & 1.33 cde \\
\hline \multicolumn{5}{|c|}{ Populations' crosses } \\
\hline $\mathrm{AOR} \times \mathrm{BAH}$ & $7.17 \mathrm{efgh}$ & $5.17 \mathrm{efgh}$ & $2.00 \mathrm{bc}$ & 1.33 cde \\
\hline $\mathrm{AOR} \times \mathrm{IGS}$ & 9.17 cdef & $4.33 \mathrm{efgh}$ & $2.50 \mathrm{bc}$ & $2.00 \mathrm{bcde}$ \\
\hline $\mathrm{AOR} \times \mathrm{IZM}$ & $11.17 \mathrm{c}$ & $6.00 \mathrm{cdef}$ & $3.00 \mathrm{bc}$ & $2.33 \mathrm{bcd}$ \\
\hline $\mathrm{AOR} \times \mathrm{MST}$ & 6.50 efghij & 3.67 efgh & $2.33 \mathrm{bc}$ & 0.83 cde \\
\hline $\mathrm{IGS} \times \mathrm{BAH}$ & 4.00 hij & $3.17 \mathrm{gh}$ & $2.17 \mathrm{bc}$ & 1.17 cde \\
\hline $\mathrm{IGS} \times \mathrm{MST}$ & 5.83 fghij & 4.67 efgh & $2.67 \mathrm{bc}$ & 1.33 cde \\
\hline $\mathrm{IZM} \times \mathrm{BAH}$ & 7.67 defg & 4.50 efgh & $3.50 \mathrm{bc}$ & 1.17 cde \\
\hline $\mathrm{IZM} \times \mathrm{IGS}$ & 7.00 efghi & 6.33 cde & $3.00 \mathrm{bc}$ & $0.33 \mathrm{e}$ \\
\hline $\mathrm{IZM} \times \mathrm{MST}$ & 7.00 efghi & $5.00 \mathrm{efgh}$ & $2.50 \mathrm{bc}$ & 1.50 cde \\
\hline $\mathrm{MST} \times \mathrm{BAH}$ & $3.50 \mathrm{j}$ & $2.50 \mathrm{~h}$ & $1.83 \mathrm{c}$ & $0.67 \mathrm{de}$ \\
\hline $\mathrm{SHH} \times \mathrm{AOR}$ & $3.67 \mathrm{ij}$ & $5.17 \mathrm{efgh}$ & $2.17 \mathrm{bc}$ & 1.17 cde \\
\hline $\mathrm{SHH} \times \mathrm{BAH}$ & 3.83 hij & $3.33 \mathrm{fgh}$ & $1.67 \mathrm{c}$ & $0.67 \mathrm{de}$ \\
\hline $\mathrm{SHH} \times \mathrm{IGS}$ & 6.17 fghij & $5.00 \mathrm{efgh}$ & $2.83 \mathrm{bc}$ & 1.00 cde \\
\hline $\mathrm{SHH} \times \mathrm{IZM}$ & 4.50 ghij & 3.83 efgh & $2.00 \mathrm{bc}$ & 1.67 bcde \\
\hline $\mathrm{SHH} \times \mathrm{MST}$ & $3.67 \mathrm{ij}$ & $2.83 \mathrm{~h}$ & $1.17 \mathrm{c}$ & $0.33 \mathrm{e}$ \\
\hline \multicolumn{5}{|c|}{ Checks } \\
\hline EPS20 & . & $5.00 \mathrm{efgh}$ & $1.67 \mathrm{c}$ & $3.33 \mathrm{ab}$ \\
\hline EPS20 $\times$ EPS21 & $19.00 \mathrm{a}$ & $11.50 \mathrm{a}$ & $7.17 \mathrm{a}$ & 1.83 bcde \\
\hline EPS21 & $15.50 \mathrm{~b}$ & $8.67 \mathrm{bc}$ & $7.17 \mathrm{a}$ & $2.50 \mathrm{abc}$ \\
\hline $\mathrm{EP} 17 \times \mathrm{EP} 42$ & . & 4.67 efgh & $4.80 \mathrm{ab}$ & $4.17 \mathrm{a}$ \\
\hline Means & 6.59 & 4.99 & 2.83 & 1.59 \\
\hline $\operatorname{LSD}_{(0.05)}$ & 3.37 & 2.75 & 2.85 & 1.76 \\
\hline
\end{tabular}


Table 1. Cont.

\begin{tabular}{|c|c|c|c|c|}
\hline \multicolumn{5}{|c|}{ Grain Yield (t/ha) } \\
\hline & \multicolumn{2}{|c|}{ Water Stress } & \multicolumn{2}{|c|}{ Control } \\
\hline & Without N & With N & Without N & With $\mathbf{N}$ \\
\hline \multicolumn{5}{|c|}{ Populations } \\
\hline AOR & 0.52 ghi & 1.04 bcdef & $1.98 \mathrm{fgh}$ & 3.16 ghij \\
\hline BAH & 0.79 cdefgh & 1.04 bcdef & 2.55 cdefg & 3.54 efghi \\
\hline IGS & 0.67 defgh & 0.63 defghi & $3.10 \mathrm{abcd}$ & 3.51 efghi \\
\hline $\mathrm{IZM}$ & $0.49 \mathrm{hi}$ & 0.52 efghi & 1.70 ghi & $2.81 \mathrm{ij}$ \\
\hline MST & $0.47 \mathrm{hi}$ & 0.48 fghi & 2.06 efgh & $2.24 \mathrm{j}$ \\
\hline $\mathrm{SHH}$ & $0.59 \mathrm{fgh}$ & 0.64 defghi & 2.62 cdefg & $2.70 \mathrm{ij}$ \\
\hline \multicolumn{5}{|c|}{ Populations' crosses } \\
\hline $\mathrm{AOR} \times \mathrm{BAH}$ & $1.04 \mathrm{abcd}$ & 1.12 abcde & 2.80 abcdef & 3.75 defgh \\
\hline $\mathrm{AOR} \times \mathrm{IGS}$ & 0.95 bcdef & $1.69 \mathrm{a}$ & $3.81 \mathrm{a}$ & $4.89 \mathrm{bc}$ \\
\hline $\mathrm{AOR} \times \mathrm{IZM}$ & 0.71 defgh & $1.38 \mathrm{abc}$ & $3.38 \mathrm{abc}$ & 3.80 defgh \\
\hline $\mathrm{AOR} \times \mathrm{MST}$ & $0.65 \mathrm{efgh}$ & 0.64 defghi & 3.09 abcde & 3.81 defgh \\
\hline $\mathrm{IGS} \times \mathrm{BAH}$ & 0.83 cdefgh & $1.47 \mathrm{ab}$ & 2.70 bcdefg & 4.23 bcdef \\
\hline $\mathrm{IGS} \times \mathrm{MST}$ & 0.75 cdefgh & 1.00 bcdefg & $3.09 \mathrm{abcd}$ & 4.06 cdefg \\
\hline $\mathrm{IZM} \times \mathrm{BAH}$ & 1.00 abcde & 0.77 cdefghi & 2.83 abcdef & $4.62 \mathrm{bcd}$ \\
\hline $\mathrm{IZM} \times \mathrm{IGS}$ & 0.78 cdefgh & 0.67 defghi & $3.70 \mathrm{ab}$ & $4.92 \mathrm{bc}$ \\
\hline $\mathrm{IZM} \times \mathrm{MST}$ & 0.82 cdefgh & 0.95 bcdefghi & $3.16 \mathrm{abcd}$ & 3.44 fghi \\
\hline $\mathrm{MST} \times \mathrm{BAH}$ & 0.66 defgh & 0.97 bcdefgh & 2.50 cdefg & 3.09 hij \\
\hline $\mathrm{SHH} \times \mathrm{AOR}$ & $1.31 \mathrm{ab}$ & 0.73 defghi & $3.10 \mathrm{abcd}$ & 4.01 cdefgh \\
\hline $\mathrm{SHH} \times \mathrm{BAH}$ & 0.93 bcdef & 1.14 abcde & 3.06 abcde & 4.42 bcde \\
\hline $\mathrm{SHH} \times \mathrm{IGS}$ & 0.87 cdefg & $1.23 \mathrm{abcd}$ & $3.74 \mathrm{a}$ & $5.09 \mathrm{ab}$ \\
\hline $\mathrm{SHH} \times \mathrm{IZM}$ & $1.12 \mathrm{abc}$ & $1.24 \mathrm{abcd}$ & 3.18 abcd & 4.30 bcdef \\
\hline $\mathrm{SHH} \times \mathrm{MST}$ & 0.71 defgh & $1.24 \mathrm{abcd}$ & 2.89 abcdef & 4.14 cdef \\
\hline \multicolumn{5}{|c|}{ Checks } \\
\hline EPS20 & $0.06 \mathrm{j}$ & $0.36 \mathrm{hi}$ & $0.73 \mathrm{i}$ & $0.70 \mathrm{k}$ \\
\hline EPS20 $\times$ EPS21 & 0.51 ghi & 0.65 defghi & $2.32 \mathrm{defg}$ & 3.92 defgh \\
\hline EPS21 & $0.17 \mathrm{ij}$ & 0.40 ghi & $1.09 \mathrm{hi}$ & $2.70 \mathrm{ij}$ \\
\hline $\mathrm{EP} 17 \times \mathrm{EP} 42$ & $1.35 \mathrm{a}$ & $0.32 \mathrm{i}$ & $3.80 \mathrm{a}$ & $5.94 \mathrm{a}$ \\
\hline Means & 0.77 & 0.91 & 2.73 & 3.73 \\
\hline $\operatorname{LSD}_{(0.05)}$ & 0.38 & 0.63 & 1.03 & 0.93 \\
\hline
\end{tabular}

a Means followed by the same letter in the row are not significantly different at $p$-value of 0.05 ; $^{\mathrm{b}}$ anthesis-silking interval; ${ }^{c}$ nitrogen. LSD, Least Significant Difference.

The grain yield of SHH $\times$ IGS $(5.09 \mathrm{t} / \mathrm{ha}$ ) was not significantly different from EP17 $\times$ EP42 under optimum conditions, and was higher than the yield of the other checks (Table 1). IZM $\times$ IGS (4.92 t/ha) and AOR $\times$ IGS (4.89 t/ha) and four other crosses were not significantly different from SHH $\times$ IGS. Under no-nitrogen supply, besides EP17 $\times$ EP42 $(3.80 \mathrm{t} / \mathrm{ha})$ and AOR $\times$ IGS $(3.81 \mathrm{t} / \mathrm{ha})$, most crosses involving IGS and the population IGS (3.10 t/ha) were among the most yielding genotypes. Conversely, under wa- 
ter stress, AOR $\times$ IGS $(1.69 \mathrm{t} / \mathrm{ha})$ had the highest yield with nitrogen, followed by IGS $\times$ BAH (1.47 t/ha) and crosses involving SHH with BAH (1.14 t/ha), IGS (1.23 t/ha), $\operatorname{IZM}(1.24 \mathrm{t} / \mathrm{ha})$, or MST (1.24 t/ha), while EP17 $\times$ EP42 had the lowest yield. Finally, under water stress and no-nitrogen, EP17 $\times$ EP42 (1.35 t/ha), SHH $\times$ AOR (1.31 t/ha), and three more crosses had the highest yield.

\subsection{Varietal and Heterosis Effects among Algerian Maize Populations}

Analysis of diallel crosses was made separately for each treatment (Table 2, Supplementary Table S5). Combined analyses over years revealed significant differences among years under well-watered conditions and both nitrogen rates. Entries were significantly different for all treatments except for yield under water stress with no-nitrogen supply. Under well-watered conditions, varietal effect was significant for plant height (under both nitrogen rates) and yield (with nitrogen supply). Under well-watered conditions, heterosis was significant for early vigor and yield (under nitrogen supply) and for all traits except ASI (under no-nitrogen supply). Average heterosis was significant for early vigor and grain yield under well-watered conditions with nitrogen. Varietal heterosis was significant under water stress for ASI (under nitrogen supply), early vigor (under no-nitrogen supply), and for grain yield under well-watered conditions with nonitrogen supply. Average heterosis was significant and positive for all traits under all treatments, except ASI under nitrogen stress, indicating the existence of the heterosis in this set of diallel crosses (Table 3 and Supplementary Table S6). For early vigor, the most favorable populations under water stress conditions were IZM (under no-nitrogen supply) and IGS (under nitrogen supply) with positive and significant varietal heterosis (0.53 and 0.46 , respectively).

Table 2. Mean squares of ASI and grain yield from the Analysis II of Gardner and Eberhart [42] of the diallel made with six Algerian maize populations evaluated, along with four checks in two years, in Algiers, under drought and nitrogen deficiency.

\begin{tabular}{|c|c|c|c|c|c|}
\hline \multicolumn{6}{|c|}{ Well-Watered } \\
\hline & & \multicolumn{2}{|c|}{ With Nitrogen } & \multicolumn{2}{|c|}{ Without Nitrogen } \\
\hline Sources of Variation & df & $\begin{array}{l}\text { ASI }^{a} \\
\text { (days) }\end{array}$ & $\begin{array}{c}\text { Grain Yield } \\
\text { (t/ha) }\end{array}$ & $\begin{array}{c}\text { ASI } \\
\text { (days) }\end{array}$ & $\begin{array}{c}\text { Grain Yield } \\
(\mathrm{t} / \mathrm{ha})\end{array}$ \\
\hline Year & 1 & $4.57 *$ & $2.51 *$ & $26.53^{* * *}$ & $1.96^{\mathrm{ns}}$ \\
\hline Rep (Year) & 4 & $4.92 * *$ & $0.73^{\mathrm{ns}}$ & $11.76^{* * *}$ & $7.04^{* * *}$ \\
\hline Entry & 20 & $2.28 *$ & $3.49 * * *$ & $2.69 *$ & $1.85^{* * *}$ \\
\hline Variety & 5 & $3.66^{\mathrm{ns}}$ & $3.96 * *$ & $7.94^{\mathrm{ns}}$ & $2.05^{\mathrm{ns}}$ \\
\hline Heterosis & 15 & $1.81^{\mathrm{ns}}$ & $3.33^{* * *}$ & $0.84^{\mathrm{ns}}$ & $1.77^{*}$ \\
\hline Average heterosis & 1 & $5.73^{\mathrm{ns}}$ & $35.67^{* *}$ & $0.53^{\mathrm{ns}}$ & $16.38^{\mathrm{ns}}$ \\
\hline Variety heterosis & 5 & $1.23^{\mathrm{ns}}$ & $1.47^{\mathrm{ns}}$ & $0.28^{\mathrm{ns}}$ & $1.51 *$ \\
\hline Specific heterosis & 9 & $1.70^{\mathrm{ns}}$ & $0.77^{\mathrm{ns}}$ & $1.20^{\mathrm{ns}}$ & $0.28^{\mathrm{ns}}$ \\
\hline Year $\times$ Entry & 20 & $1.19^{\mathrm{ns}}$ & $0.40^{\mathrm{ns}}$ & $1.82^{\mathrm{ns}}$ & $0.58^{\mathrm{ns}}$ \\
\hline Variety $\times$ Year & 5 & $0.96^{\mathrm{ns}}$ & $0.34^{\mathrm{ns}}$ & $2.10^{\mathrm{ns}}$ & $0.56^{\mathrm{ns}}$ \\
\hline Heterosis $\times$ Year & 15 & $1.26^{\mathrm{ns}}$ & $0.41^{\mathrm{ns}}$ & $1.57^{\mathrm{ns}}$ & $0.63^{\mathrm{ns}}$ \\
\hline Average heterosis $\times$ Year & 1 & $2.40^{\mathrm{ns}}$ & $0.00^{\mathrm{ns}}$ & $1.02^{\mathrm{ns}}$ & $0.74^{\mathrm{ns}}$ \\
\hline Variety heterosis $\times$ Year & 5 & $0.81^{\mathrm{ns}}$ & $0.50^{\mathrm{ns}}$ & $2.74^{\mathrm{ns}}$ & $0.26^{\mathrm{ns}}$ \\
\hline Specific heterosis $\times$ Year & 9 & $1.39^{\mathrm{ns}}$ & $0.41^{\mathrm{ns}}$ & $1.05^{\mathrm{ns}}$ & $0.82^{\mathrm{ns}}$ \\
\hline \multicolumn{6}{|l|}{ Error } \\
\hline Degrees of freedom & & 80 & 80 & 79 & 79 \\
\hline Mean squares & & 1.16 & 0.59 & 1.46 & 0.601 \\
\hline
\end{tabular}


Table 2. Cont.

\begin{tabular}{|c|c|c|c|c|c|}
\hline \multicolumn{6}{|c|}{ Water-Stressed } \\
\hline & & \multicolumn{2}{|c|}{ With Nitrogen } & \multicolumn{2}{|c|}{ Without Nitrogen } \\
\hline Sources of Variation & df & $\begin{array}{c}\text { ASI } \\
\text { (days) }\end{array}$ & $\begin{array}{l}\text { Yield } \\
\text { (t/ha) }\end{array}$ & $\begin{array}{c}\text { ASI } \\
\text { (days) }\end{array}$ & $\begin{array}{c}\text { Grain Yield } \\
(\mathrm{t} / \mathrm{ha})\end{array}$ \\
\hline Year & 1 & $42.00 *$ & $0.19^{\mathrm{ns}}$ & $95.98^{* * *}$ & $0.27^{\mathrm{ns}}$ \\
\hline Rep (Year) & 4 & $27.80^{* *}$ & $4.66^{* * *}$ & $35.27^{* *}$ & $2.07^{* * *}$ \\
\hline Entry & 20 & $13.91^{* *}$ & $0.66 * *$ & $31.81^{* * *}$ & $0.27^{\mathrm{ns}}$ \\
\hline Variety & 5 & $25.96^{* *}$ & $0.41 *$ & $92.28 *$ & $0.25^{\mathrm{ns}}$ \\
\hline Heterosis & 15 & $12.71 *$ & $0.71 *$ & $12.79 *$ & $0.28^{* *}$ \\
\hline Average heterosis & 1 & $53.41^{* *}$ & $3.17^{\mathrm{ns}}$ & $20.38^{* *}$ & $2.11^{\mathrm{ns}}$ \\
\hline Variety heterosis & 5 & $20.73^{*}$ & $0.21^{\mathrm{ns}}$ & $13.77^{\mathrm{ns}}$ & $0.16^{\mathrm{ns}}$ \\
\hline Specific heterosis & 9 & $4.14^{\mathrm{ns}}$ & $0.71^{\mathrm{ns}}$ & $11.97^{\mathrm{ns}}$ & $0.14^{\mathrm{ns}}$ \\
\hline Year $\times$ Entry & 20 & $4.11^{\mathrm{ns}}$ & $0.23^{\mathrm{ns}}$ & $6.20^{\mathrm{ns}}$ & $0.08^{\mathrm{ns}}$ \\
\hline Variety $\times$ Year & 5 & $2.11^{\mathrm{ns}}$ & $0.09 \mathrm{~ns}$ & $11.88^{\mathrm{ns}}$ & $0.08^{\mathrm{ns}}$ \\
\hline Heterosis $\times$ Year & 15 & $4.38^{\mathrm{ns}}$ & $0.29^{\mathrm{ns}}$ & $5.63^{\mathrm{ns}}$ & $0.08^{\mathrm{ns}}$ \\
\hline Average heterosis $\times$ Year & 1 & $2.06^{\mathrm{ns}}$ & $0.25^{\mathrm{ns}}$ & $5.26^{\mathrm{ns}}$ & $0.03^{\mathrm{ns}}$ \\
\hline Variety heterosis $\times$ Year & 5 & $3.22^{\mathrm{ns}}$ & $0.13^{\mathrm{ns}}$ & $3.45^{\mathrm{ns}}$ & $0.08^{\mathrm{ns}}$ \\
\hline Specific heterosis $\times$ Year & 9 & $5.23^{\mathrm{ns}}$ & $0.39^{\mathrm{ns}}$ & $7.58^{\mathrm{ns}}$ & $0.08^{\mathrm{ns}}$ \\
\hline \multicolumn{6}{|l|}{ Error } \\
\hline Degrees of freedom & & 77 & 78 & 75 & 80 \\
\hline Mean squares & & 6.16 & 0.29 & 7.36 & 0.17 \\
\hline
\end{tabular}

a Anthesis-silking interval; ${ }^{\text {ns }},{ }^{*},{ }^{* *},{ }^{* * *}$ not significant, significant at the $0.05,0.01$, and 0.001 probability levels, respectively.

For plant height, negative significant specific heterosis $(-10.99 \mathrm{~cm})$ was recorded for SHH $\times$ AOR (as for early vigor) and for AOR $\times$ IGS $(11.76 \mathrm{~cm}$ ) which led to provide taller plants under water stress and nitrogen supply conditions. Under well-watered conditions with nitrogen supply, there was a highly positive significant specific heterosis for IGS $\times$ BAH $(20.87 \mathrm{~cm})$. IGS and IZM showed a significant and positive varietal effect under well-watered conditions. Under water stress conditions with no-nitrogen supply, significant and positive varietal effect was found for BAH $(16.5 \mathrm{~cm})$. Average heterosis for this trait was significant and positive under all treatments. For ASI, significant and negative specific heterosis was found in $\mathrm{SHH} \times \mathrm{AOR}(-2.13$ days) under water stress conditions with no-nitrogen supply and IZM $\times$ IGS ( -1.13 days) under well-watered conditions with nitrogen supply. BAH had a significant and negative varietal heterosis $(-2.11$ days) in the presence of nitrogen fertilizer under water stress. Under water stress conditions with nitrogen supply, AOR ( -2.03 days) had a negative significant varietal effect. Under water-stress conditions with no-nitrogen supply, a significant negative varietal effect was found for MST (-3.49 days). Moreover, under well-watered conditions with nitrogen supply, a significant negative value for MST ( -0.97 day) was observed. All the negative values indicated that those populations provided stress tolerant plants with low ASI. Average heterosis for this trait was negative under all treatments and significant only under no-nitrogen supply. 
Table 3. Genetic parameters of ASI ${ }^{a}$ and grain yield from the analyses of Gardner and Eberhart [42] (varietal effect, heterosis effect, specific heterosis, and average heterosis) for four traits in the diallel made among six Algerian maize populations evaluated in two years, in Algiers, under both managed drought and nitrogen deficiency.

\begin{tabular}{|c|c|c|c|c|}
\hline \multicolumn{5}{|c|}{ ASI (days) } \\
\hline & \multicolumn{2}{|c|}{ Water-Stressed } & \multicolumn{2}{|c|}{ Well-Watered } \\
\hline Populations & Without $\mathbf{N}^{\mathbf{b}}$ & With N & Without N & With $\mathbf{N}$ \\
\hline \multicolumn{5}{|c|}{ Varietal effect } \\
\hline AOR & 0.18 & $-2.03 *$ & 0.33 & 0.19 \\
\hline BAH & -0.99 & $2.63^{* *}$ & 0.17 & 0.69 \\
\hline IGS & $2.85 *$ & -0.03 & 0.33 & -0.31 \\
\hline IZM & $3.76^{* *}$ & $3.63 * *$ & $1.17^{*}$ & 0.69 \\
\hline MST & $-3.49^{* *}$ & -1.67 & -0.83 & $-0.97 *$ \\
\hline $\mathrm{SHH}$ & $-2.32 *$ & $-2.53 *$ & $-1.17^{*}$ & -0.31 \\
\hline \multicolumn{5}{|c|}{ Varietal heterosis } \\
\hline AOR & $1.76^{*}$ & $1.64 *$ & -0.11 & 0.36 \\
\hline $\mathrm{BAH}$ & -0.53 & $-2.11^{* *}$ & -0.24 & -0.56 \\
\hline IGS & -0.95 & 0.43 & 0.18 & 0.15 \\
\hline IZM & -0.12 & -0.86 & -0.03 & -0.06 \\
\hline MST & 0.80 & 0.04 & 0.10 & 0.19 \\
\hline $\mathrm{SHH}$ & -0.95 & 0.85 & 0.10 & -0.10 \\
\hline \multicolumn{5}{|c|}{ Specific heterosis } \\
\hline $\mathrm{AOR} \times \mathrm{BAH}$ & 0.29 & 0.97 & -0.26 & -0.08 \\
\hline AOR $\times$ IGS & 0.79 & -1.08 & -0.26 & 0.38 \\
\hline $\mathrm{AOR} \times \mathrm{IZM}$ & 1.50 & 0.05 & 0.03 & 0.42 \\
\hline $\mathrm{AOR} \times \mathrm{MST}$ & -0.46 & -0.53 & 0.24 & -0.50 \\
\hline $\mathrm{IGS} \times \mathrm{BAH}$ & -1.50 & -0.83 & -0.38 & 0.21 \\
\hline IGS $\times$ MST & 0.25 & 0.68 & 0.28 & 0.46 \\
\hline $\mathrm{IZM} \times \mathrm{BAH}$ & -0.88 & 0.03 & -0.74 & 0.08 \\
\hline $\mathrm{IZM} \times \mathrm{IGS}$ & -1.29 & 0.59 & -0.26 & $-1.13^{* *}$ \\
\hline $\mathrm{IZM} \times \mathrm{MST}$ & 0.13 & 0.47 & -0.09 & 0.33 \\
\hline $\mathrm{MST} \times \mathrm{BAH}$ & -0.58 & -0.28 & -0.05 & 0.00 \\
\hline $\mathrm{SHH} \times \mathrm{AOR}$ & $-2.13 *$ & 0.59 & 0.24 & -0.21 \\
\hline $\mathrm{SHH} \times \mathrm{BAH}$ & 0.92 & 0.18 & -0.05 & -0.04 \\
\hline $\mathrm{SHH} \times \mathrm{IGS}$ & 1.75 & 0.63 & 0.62 & 0.08 \\
\hline $\mathrm{SHH} \times \mathrm{IZM}$ & -1.21 & -1.08 & -0.43 & 0.46 \\
\hline $\mathrm{SHH} \times \mathrm{MST}$ & 0.67 & -0.33 & -0.38 & -0.29 \\
\hline Average heterosis & -0.93 & $-1.50 * *$ & -0.14 & $-0.47^{*}$ \\
\hline
\end{tabular}


Table 3. Cont.

\begin{tabular}{|c|c|c|c|c|}
\hline \multicolumn{5}{|c|}{ Grain Yield (t/ha) } \\
\hline & \multicolumn{2}{|c|}{ Water-Stressed } & \multicolumn{2}{|c|}{ Well-Watered } \\
\hline & Without N & With N & Without N & With N \\
\hline \multicolumn{5}{|c|}{ Varietal effect } \\
\hline AOR & -0.07 & 0.32 & -0.35 & 0.16 \\
\hline $\mathrm{BAH}$ & 0.21 & 0.32 & 0.22 & $0.55 *$ \\
\hline IGS & 0.08 & -0.10 & $0.77^{*}$ & 0.51 \\
\hline $\mathrm{IZM}$ & -0.10 & -0.20 & $-0.64^{*}$ & -0.18 \\
\hline MST & -0.12 & -0.24 & -0.27 & $-0.75^{* *}$ \\
\hline $\mathrm{SHH}$ & 0.00 & -0.09 & 0.28 & -0.29 \\
\hline \multicolumn{5}{|c|}{ Varietal heterosis } \\
\hline AOR & 0.10 & -0.12 & 0.30 & -0.23 \\
\hline BAH & -0.08 & -0.14 & $-0.56^{*}$ & $-0.46^{*}$ \\
\hline IGS & -0.09 & 0.21 & -0.04 & 0.33 \\
\hline $\mathrm{IZM}$ & 0.06 & 0.00 & $0.46^{*}$ & 0.15 \\
\hline MST & -0.14 & -0.03 & -0.10 & -0.20 \\
\hline $\mathrm{SHH}$ & 0.14 & 0.09 & -0.07 & $0.42 *$ \\
\hline \multicolumn{5}{|c|}{ Specific heterosis } \\
\hline $\mathrm{AOR} \times \mathrm{BAH}$ & 0.07 & -0.01 & -0.02 & -0.08 \\
\hline AOR $\times$ IGS & 0.05 & $0.41 *$ & 0.21 & 0.29 \\
\hline $\mathrm{AOR} \times \mathrm{IZM}$ & -0.25 & 0.37 & -0.02 & -0.28 \\
\hline $\mathrm{AOR} \times \mathrm{MST}$ & -0.10 & -0.33 & 0.06 & 0.37 \\
\hline $\mathrm{IGS} \times \mathrm{BAH}$ & -0.02 & 0.21 & -0.33 & -0.34 \\
\hline IGS $\times$ MST & 0.12 & -0.09 & -0.15 & -0.12 \\
\hline $\mathrm{IZM} \times \mathrm{BAH}$ & -0.09 & 0.23 & 0.00 & $-0.58 *$ \\
\hline $\mathrm{IZM} \times \mathrm{IGS}$ & -0.06 & $-0.47^{*}$ & 0.08 & 0.11 \\
\hline $\mathrm{IZM} \times \mathrm{MST}$ & 0.13 & 0.12 & 0.12 & -0.20 \\
\hline $\mathrm{MST} \times \mathrm{BAH}$ & -0.04 & 0.03 & 0.05 & -0.32 \\
\hline $\mathrm{SHH} \times \mathrm{AOR}$ & 0.22 & $-0.43 *$ & -0.24 & -0.29 \\
\hline $\mathrm{SHH} \times \mathrm{BAH}$ & -0.11 & 0.00 & 0.30 & 0.16 \\
\hline $\mathrm{SHH} \times \mathrm{IGS}$ & -0.09 & -0.06 & 0.19 & 0.06 \\
\hline $\mathrm{SHH} \times \mathrm{IZM}$ & 0.09 & 0.22 & -0.17 & -0.21 \\
\hline $\mathrm{SHH} \times \mathrm{MST}$ & -0.11 & 0.27 & -0.08 & 0.27 \\
\hline Average heterosis & $0.29 * *$ & 0.36 ** & $0.80^{* * *}$ & $1.18^{* * *}$ \\
\hline
\end{tabular}

a Anthesis-silking interval; ${ }^{\mathrm{b}}$ nitrogen; ${ }^{*},{ }^{* *},{ }^{* * *}$ significant at the $0.05,0,01$ and 0.001 probability levels, respectively

Concerning yield, AOR $\times$ IGS had a positive significant specific heterosis $(0.41 \mathrm{t} / \mathrm{ha})$ under water-stress conditions with nitrogen supply. Contrarily, IZM $\times$ IGS $(-0.47 \mathrm{t} / \mathrm{ha})$ and $\mathrm{SHH} \times \mathrm{AOR}(-0.43 \mathrm{t} / \mathrm{ha})$ exhibited significant and negative specific heterosis. Under well-watered conditions with nitrogen supply, IZM $\times$ BAH showed negative specific heterosis $(-0.58 \mathrm{t} / \mathrm{ha})$. IZM and SHH showed a significant positive varietal heterosis ( $0.46 \mathrm{t} /$ ha with no-nitrogen supply) and (0.42 $\mathrm{t} / \mathrm{ha}$ with nitrogen supply), respectively. Contrarily, under the same water regime, $\mathrm{BAH}$ had the least varietal heterosis values: $(-0.46 \mathrm{t} / \mathrm{ha})$ with nitrogen supply and $(-0.56 \mathrm{t} / \mathrm{ha})$ with no-nitrogen supply. Under 
well-watered conditions, varietal effect was significant and positive for BAH (0.55 $\mathrm{t} / \mathrm{ha}$ with nitrogen supply) and IGS (0.77 t/ha with no-nitrogen supply), whilst significant and negative varietal effect for IZM $(-0.64 \mathrm{t} / \mathrm{ha})$ with no-nitrogen supply and for MST $(-0.75 \mathrm{t} / \mathrm{ha})$ with nitrogen supply. Average heterosis for this trait was very significant and positive for all treatments.

\section{Discussion}

There was large variability within the Algerian maize populations per se, as well as among populations' crosses both under control and stress conditions (Supplementary Table S2). Among populations per se, yield variation was minimum under control conditions, ranging from $2.24 \mathrm{t} /$ ha for MST to 3.54 for BAH, and maximum for water stress with nitrogen fertilization, as the grain yield of AOR and BAH doubled that of MST and IZM (Table 1). The ranges of variability increased among population crosses under water stress, as the yield of $\mathrm{SHH} \times \mathrm{AOR}$ duplicated that of AOR $\times$ MST and MST $\times \mathrm{BAH}$ under combined stresses; though ranges of variation for yield decreased under irrigation (Table 1). Furthermore, the ranges of variation were even larger for ASI under water stress, being 3.50 days for MST and 10.75 for IZM, while among hybrids, MST $\times$ BAH had 3.50 days and AOR $\times$ IZM 11.17 under combined stresses. Moreover, variation for ASI was narrower under irrigation. This genetic variation makes selection possible for releasing high-performance hybrids [44]. Our results were in accordance with Cherchali et al. [40], who reported large variability among Algerian populations and their crosses. Furthermore, significant genetic variability under stress conditions is of the utmost importance for progress from selection for improved grain yield under drought stress and low nitrogen $[45,46]$. That variability could also be useful for breeding programs in temperate environments because Algerian maize has a high degree of genetic diversity and a wide adaptability to temperate regions [32,33].

Chiuta and Mutengwa [47] reported that the genetic material used in breeding programs for crop improvement must undergo a rigorous screening process to determine its inherent potential because their success depends on genetic material ability to transmit the desired characteristics to its offspring. Our agronomic data showed that early vigor, plant height and grain yield were superior in crosses than in populations per se. Nevertheless, differences between populations and their crosses for ASI were not as high as for the previous traits.

The lack of significant genotype $\times$ year interaction for all the traits under all the treatments is evidence of the stability of genotypes across years (Supplementary Table S2). This result is consistent with the finding of Oyekale et al. [48], who found that the response patterns of the genotypes were similar for the measured traits in the diverse environments. Significant genotype $\times$ environment interaction would reduce selection progress in breeding programs and make it difficult to select stable and high-performant genotypes at various locations [49].

According to per se performance, BAH was tolerant to nitrogen stress, based on early vigor and ASI, and IGS based on early vigor and yield because both improved its relative performance under no-nitrogen fertilization, compared to standard nitrogen fertilization (Table 1 and Supplementary Table S4). BAH belongs to the genetic group III, while IGS belongs to the genetic group I, defined by Cherchali et al. [40], and these populations come from different regions. Concerning populations' crosses, those with better rank in yield and plant height under no-nitrogen fertilization and drought than under control conditions were $\mathrm{IZM} \times \mathrm{BAH}$ and $\mathrm{SHH} \times \mathrm{IZM}$, as both crosses were not significantly different from the highest yielder cross $(\mathrm{SHH} \times \mathrm{AOR})$ for yield under combined stresses, while they significantly differed from the highest yielder cross $(\mathrm{SHH} \times \mathrm{IGS})$ for yield under control conditions (Table 1). Furthermore, IZM $\times \mathrm{BAH}$ and $\mathrm{SHH} \times \mathrm{IZM}$ also had better relative plant height under water stress than under control conditions. Besides this, AOR $\times \mathrm{BAH}$ and $\mathrm{SHH} \times \mathrm{AOR}$ had better relative yield under no-nitrogen fertilization and drought than under control conditions. However, none of the checks had better performance under 
nitrogen or drought stress than under control conditions. Therefore, we could choose the best performant parents and specially the most promising crosses as base of future breeding programs.

For early vigor, the contribution of heterosis variances was higher under all treatments (Supplementary Table S5). The heterosis mean squares are superior to the varietal effect mean squares, which mean that for most traits, the role of non-additive genes is more important than the role of additive genes, and heterosis is the main component that explains the differences between entries being the varietal effect important only under water stress. For plant height, varietal heterosis and specific heterosis presented non-significant mean squares under all treatments. Furthermore, the varietal effects explained the highest proportion of the sum of squares under well-watered with nitrogen supply, indicating that this trait is mainly controlled by additive effects. These findings are consistent with the results of Zeleke et al. [44], who reported that additive gene action was more important than non-additive gene action in the inheritance of plant height. Contrarily to our results, under low nitrogen, Noëlle et al. [50] reported that plant height was influenced mainly by additive gene effects. Ribeiro et al. [51] reported non-significant specific heterosis for plant height indicating that non-additive gene effects were not important in the inheritance of this trait under low nitrogen.

For ASI, varietal effects and heterosis were significant only under water stress, suggesting that both additive and non-additive genetic action were important for inheritance of this trait under stress (Table 2). Accordingly, Amegbor et al. [52] reported the preponderance of SCA over GCA for ASI under managed drought. They suggested that substantial genetic enhancement could be achieved by using breeding programs that capitalize on the effects of non-additive genes, such as hybridization. However, under water stress with no-nitrogen supply, varietal effect presented higher proportion of the sum of squares $(45 \%)$ than the heterosis effect (19\%), which indicated that additive genetic effects controlled ASI. Alphonse et al. [53] reported that, under drought stress and lownitrogen conditions, secondary traits (such as ASI) might bring promising genetic gains. This secondary trait can be used to select for stress tolerance, because their heritability under stress remains high and their genetic correlation with grain yield increased.

For yield, heterosis effect was significant under all treatments (Table 2). The sum of squares of genotypes (entries) explained most of the variability, while years or the genotype $\times$ year interaction had minor contributions. Within the genotypic variation, the heterosis contribution to the total variation was 50\% larger than the average heterosis, and several times larger than the varietal effect under control conditions, though differences in proportion of variance explained among sources of variation were lower under no-nitrogen fertilization. Similarly, under managed drought stress conditions, Umar et al. [54] and Njeri et al. [55] reported non-additive gene action for this trait over additive gene effects. Meseka et al. [56] and Noëlle et al. [50] also reported the predominance of non-additive genetic effects for grain yield suggesting that hybrid development could be interesting under no-nitrogen fertilization in order to exploit non-additive gene effects. Contrarily to our results, Alphonse et al. [53] proposed that in most environments, the effect of additive genes is the most important factor in the genetic variation controlling grain yield. In the same way, Beck et al. [57] reported the predominance of GCA to SCA indicating the relative importance of additive gene action respect to non-additive gene action for this trait. Furthermore, in all tested environments of the study of Badu-Apraku et al. [58], more than 60\% of the total genetic effects was attributed to GCA for grain yield. In addition, Makumbi [59], Amegbor et al. [52], Chiuta and Mutengwa [47], and Ribeiro et al. [51] reported the major role of additive effects under optimal conditions. Besides this, Ribeiro et al. [51] and Oyekale et al. [48] reported this result under low nitrogen, and Amegbor et al. [52] and Oyetunde et al. [60] reported it under drought among maize populations. Varietal effect for yield was significant under nitrogen supply under well-watered and under water stress, while it was not significant under no-nitrogen fertilization, indicating that there is genetic diversity for yield and, particularly, under drought stress (Table 2). Heterosis was 
significant for yield under all treatments, but varietal heterosis for yield was significant only under well-watered with no-nitrogen supply, implying that capitalizing heterosis in breeding programs is not very promising under drought conditions or when nitrogen is available at normal rates. Furthermore, this indicated that yield is controlled by the two types of gene action. The important role of the two gene actions in conferring drought tolerance is emphasized by Oyetunde et al. [60], who found that both additive and nonadditive genetic effects were important in the inheritance of grain yield with high nitrogen fertilization. In addition, Ribeiro et al. [51] confirmed that the presence of the two types of gene action under low nitrogen could increase yield through selection. According to Zeleke et al. [44], the breeding implication of the importance of both additive and nonadditive gene actions is the use of reciprocal recurrent selection, which is useful for the genetic improvement of the population traits.

Average heterosis was significant and positive under most treatments, indicating that the crosses were, on average, greater than the populations per se for all traits (Table 3 and Supplementary Table S6). For early vigor, under water stress conditions, significant and positive varietal heterosis effect was found for IZM under no-nitrogen supply and IGS under nitrogen supply (Supplementary Table S6). For plant height, positive and significant variety effects were detected for BAH under water and nitrogen stress, IGS under well-watered with or without nitrogen supply, and IZM under control conditions. For ASI, significant and negative variety effects were identified for AOR under drought, MST under water and nitrogen stress and also under control conditions, and SHH under all stress conditions but not under control conditions, and varietal heterosis for BAH under drought (Table 3).

For yield, there were positive and significant variety effects for BAH under control conditions, and IGS under nitrogen stress, while IZM and MST had negative variety effects under control and nitrogen stress, respectively (Table 3). Varietal heterosis was significant and positive for IZM under nitrogen stress and SHH under control conditions, and negative and significant for $\mathrm{BAH}$ under both nitrogen treatments with irrigation. However, none of the populations had significant variety effects or varietal heterosis for grain yield under drought stress, irrespective of the nitrogen treatment. These IGS and IZM have high probability of transmitting alleles for tolerance for nitrogen tolerance to their progenies, as reported by Amegbor et al. [52]. None of the crosses had significant positive specific heterosis for early vigor (Supplementary Table S6). For plant height, under water-stress conditions with nitrogen supply, the most promising crosses were AOR $\times$ IGS, with a significant and positive specific heterosis, and IGS $\times$ BAH under control conditions, thus implying that it could be promising for developing drought-tolerant hybrids through reciprocal recurrent selection to improve plant height. For ASI, the most promising crosses identified as excellent specific combiners were $\mathrm{SHH} \times \mathrm{AOR}$ under water stress conditions with no-nitrogen supply and IZM $\times$ IGS under optimum conditions (Table 3). For yield, under water stress conditions with nitrogen supply, the most promising cross, based on specific heterosis, was AOR $\times$ IGS. On the other hand, with nitrogen fertilizatioin, IZM $\times$ IGS and SHH $\times$ AOR had negative specific heterosis for yield under water stress and IZM $\times$ BAH under irrigation. Conversely, specific heterosis for yield was no significant with no-nitrogen fertilization. Therefore, none of the population crosses was a promising base population for improving yield under combined stress or under low nitrogen stress, but AOR $\times$ IGS could be used in a breeding program for improving tolerance to drought, in accordance with Cherchali et al. [40], who determined that AOR $\times$ IGS is the most promising cross under well-watered conditions and suggested that the yield increase is due to the fact that both populations come from two different groups, based on genetic clusters [33], and both presented flint kernels. Adequate genetic variability is critical to the selection progress of breeding programs to increase grain yield [46]. Zeleke et al. [44] stated that crosses with a higher value of SCA effects, which also showed higher values of mean grain yield, could be effectively exploited in a hybrid breeding program. However, the parents of AOR $\times$ IGS had low and insignificant varietal heterosis, which demonstrate 
the importance of non-additive gene effects in influencing yield potential by exploiting maximum heterosis. Mogesse et al. [46] confirmed that good specific combiners were not necessarily found from the two good general combiner crosses.

\section{Conclusions}

Algerian maize populations showed large diversity for performance under stress, as well as for their possible contributions to water- and nitrogen-stress-tolerance crosses. Given all results and focusing on yield, we propose a reciprocal recurrent selection program, to take advantage of additive and non-additive effects, using AOR and IGS varieties as base material, since they showed good performance in optimum and stress conditions (except AOR under well-watered and no-nitrogen supply), with the goal of improving yield heterosis for AOR $\times$ IGS cross. It is not expected to have negative effects on plant height, ASI, or early vigor. In addition, these populations, together with BAH, should be suitable materials to obtain inbred lines tolerant to drought and low nitrogen concentration.

There was no clear pattern of relationship between origin and genetic group and per se performance, parental contribution, or hybrid performance under stress conditions. Overall, these populations and crosses could be used as heterotic groups among Algerian populations for breeding programs focusing on water- or nitrogen-stress tolerance.

Supplementary Materials: The following are available online at https:/ /www.mdpi.com/2073-4 395/11/3/492/s1, Supplementary Table S1: Name and origin of the six Algerian populations used in the diallel mating design; Supple-mentary Table S2: Mean squares from the analysis of variance combined across years for four agronomic traits analyzed in a diallel among with six Algerian maize popula-tions evaluated along with four checks in two years in Algiers under drought and ni-trogen deficiency; Supplementary Table S3: Mean squares from the analysis of vari-ance of four agronomic traits analyzed in the diallel systems with six Algerian maize populations evaluated along with four checks in two years in Algiers under both managed drought and nitrogen deficiency; Supplementary Table S4: Means a three ag-ronomic traits analyzed in the diallel systems with six Algerian maize populations evaluated along with four checks in two years in Algiers under both managed drought and nitrogen deficiency; Supplementary Table S5: Mean squares from the Analysis II of Gardner and Eberhart [42] of the diallel made among with six Algerian maize popula-tions evaluated along with four checks in two years in Algiers under drought and ni-trogen deficiency; Supplementary Table S6: Genetic parameters from the analyses of Gardner and Eberhart [42] (Varietal effect, Heterosis effect, specific heterosis and av-erage heterosis) for four traits in the diallel made among six Algerian maize popula-tions evaluated in two years in Algiers under both managed drought and nitrogen de-ficiency.

Author Contributions: M.R., data recording and draft preparation; P.R., manuscript correction and final redaction; O.M., data recording and draft preparation; R.A.M., data analyses; A.D., conceptualization, materials, experimental design, data recording and analyses, and draft preparation and redaction. All authors have read and agreed to the published version of the manuscript.

Funding: This research was funded by École Nationale Supérieure Agronomique d'Alger, and the Spanish Ministerio de Innovación y Universidades (MCIU), the Agencia Estatal de Investigación (AEI) and the European Fund for Regional Development (FEDER), UE (project code PID2019-108127RB-I00).

Institutional Review Board Statement: Not applicable.

Informed Consent Statement: Not applicable.

Data Availability Statement: Data available from authors upon request.

Conflicts of Interest: The authors declare no conflict of interest.

\section{References}

1. Heinz, R.; Ribeiro, L.P.; Gonçalves, M.C.; Bhering, L.L.; Teodoro, P.E. Selection of maize top-crosses for different nitrogen levels through specific combining ability. Bragantia 2019, 78, 208-214. [CrossRef]

2. Jaidka, M.; Bathla, S.; Kaur, R. Improved Technologies for Higher Maize Production. In Maize-Production and Use; IntechOpen: London, UK, 2020. 
3. Li, K.; Ramakrishna, W. Basic Information. In Genetics, Genomics and Breeding of Maize; Wusirika, R., Bohn, M., Lai, J., Kole, C., Eds.; CRC Press: Boca Raton, FL, USA, 2014; pp. 1-13.

4. Tilman, D.; Balzer, C.; Hill, J.; Befort, B.L. Global food demand and the sustainable intensification of agriculture. Proc. Natl. Acad. Sci. USA 2011, 108, 20260-20264. [CrossRef]

5. Rabara, R.C.; Ferrer, M.C.; Jara-Rabara, J.; Sotto, R.C. Securing Diversity for Food Security: The Case of Conservation and Use of Rice Genetic Resources. In New Visions in Plant Science; Çelik, Ö., Ed.; IntechOpen: London, UK, 2018; pp. 81-96.

6. IPCC. Part A: Global and Sectoral Aspects. (Contribution of Working Group II to the Fifth Assessment Report of the Intergovernmental Panel on Climate Change); Field, C.B., Barros, V.R., Dokken, D.J., Mach, K.J., Mastrandrea, M.D., Bilir, T.E., Chatterjee, M., Ebi, K.L., Estrada, Y.O., Genova., R.C., et al., Eds.; Cambridge University Press: Cambridge, UK, 2014.

7. Mannan, M.A.; Akter Shashi, M. Amelioration of Drought Tolerance in Maize Using Rice Husk Biochar. In Maize-Production and Use; IntechOpen: London, UK, 2020; pp. 1-19.

8. Bouman, B.A.M.; Lampayan, R.M.; Tuong, T.P. Water Management in Irrigated Rice: Coping with Water Scarcity; International Rice Research Institute: Los Baños, Philippines, 2007.

9. Salgotra, R.K.; Gupta, B.B. Plant Genetic Resources and Traditional Knowledge for Food Security; Springer Nature Singapore Private Limited: Singapore, 2016.

10. Rao, G.J.N.; Reddy, J.N.; Variar, M.; Mahender, A. Molecular Breeding to Improve Plant Resistance to Abiotic Stresses. In Advances in Plant Breeding Strategies: Agronomic, Abiotic and Biotic Stress Traits; Springer International Publishing: Cham, Switzerland, 2016; pp. 283-326.

11. Mugo, S.; de Groote, H.; Bergvinson, D.; Mulaa, M.; Songa, J.; Gichuki, S. Developing Bt maize for resource-poor farmers-Recent advances in the IRMA project. Afr. J. Biotechnol. 2005, 4, 1490-1504. [CrossRef]

12. Edmeades, G.O. Drought Tolerance in Maize: An Emerging Reality; The International Service for the Acquisition of Agri-biotech Applications (ISAAA): Ithaca, NY, USA, 2008.

13. Bender, R.R.; Haegele, J.W.; Ruffo, M.L.; Below, F.E. Nutrient uptake, partitioning, and remobilization in modern, transgenic insect-protected maize hybrids. Agron. J. 2013, 105, 161-170. [CrossRef]

14. Ribeiro, P.F.; Badu-Apraku, B.; Gracen, V.E.; Danquah, E.Y.; Garcia-Oliveira, A.L.; Asante, M.D.; Afriyie-Debrah, C.; Gedil, M. Identification of quantitative trait loci for grain yield and other traits in tropical maize under high and low soil-nitrogen environments. Crop Sci. 2018, 58, 321-331. [CrossRef]

15. Al-Naggar, A.M.M.; Shafik, M.M.; Musa, R.Y.M. Multivariate Analysis of Genetic Diversity among Maize Genotypes and Trait Interrelationships under Drought and Low N Stress. In New Perspectives in Agriculture and Crop Science; Al-Naggar, D.A.M.M., Ed.; Book Publisher International (a part of SCIENCEDOMAIN International): West Bengal, India, 2020; Volume 2, pp. 70-92.

16. Masuka, B.; Araus, J.L.; Das, B.; Sonder, K.; Cairns, J.E. Phenotyping for Abiotic Stress Tolerance in Maize. J. Integr. Plant Biol. 2012, 54, 238-249. [CrossRef]

17. Dodig, D.; Božinović, S.; Nikolić, A.; Zorić, M.; Vančetović, J.; Ignjatovic-Micic, D.; Delic, N.; Weigelt-Fischer, K.; Junker, A.; Altmann, T. Image-derived traits related to mid-season growth performance of maize under nitrogen and water stress. Front. Plant Sci. 2019, 10, 814. [CrossRef]

18. Malik, W.; Dechmi, F. Modelling agricultural nitrogen losses to enhance the environmental sustainability under Mediterranean conditions. Agric. Water Manag. 2020, 230, 105966. [CrossRef]

19. Kamara, A.Y.; Ewansiha, S.U.; Tofa, A.I. Yield, N uptake and N utilization of early maturing, drought and striga-tolerant maize varieties under low N conditions. Commun. Soil Sci. Plant Anal. 2019, 50, 373-387. [CrossRef]

20. Buchanan-Wollaston, V.; Wilson, Z.; Tardieu, F.; Beynon, J.; Denby, K. Harnessing diversity from ecosystems to crops to genes. Food Energy Secur. 2017, 6, 19-25. [CrossRef]

21. Matin, M.Q.I.; Rasul, G.; Islam, A.K.M.A.; Mian, M.A.K.; Ivy, N.A.; Ahmed, J.U. Study of Genetic Diversity in Maize (Zea mays L.) Inbreds. Plant 2017, 5, 31. [CrossRef]

22. Gore, M.; Bradbury, P.; Hogers, R.; Kirst, M.; Verstege, E.; van Oeveren, J.; Peleman, J.; Buckler, E.; van Eijk, M. Evaluation of Target Preparation Methods for Single-Feature Polymorphism Detection in Large Complex Plant Genomes. Crop. Sci. 2007, 47, 135-148. [CrossRef]

23. Staller, J.E. Maize Obs and Cultures: History of Zea mays L.; Springer: Berlin/Heidelberg, Germany, 2010.

24. Aslam, M.; Maqbool, M.A.; Cengiz, R. Drought Stress in Maize (Zea mays L.). In Effects, Resistance Mechanisms, Global Achievements and Biological Strategies for Improvement; Springer International Publishing: Cham, Switzerland, 2015.

25. Hallauer, A.R.; Carena, M.J.; Filho, J.B.M. Quantitative Genetics in Maize Breeding; Springer Science+Business Media, LLC: New York, NY, USA, 2010.

26. Nelimor, C.; Badu-Apraku, B.; Nguetta, S.P.A.; Tetteh, A.Y.; Garcia-Oliveira, A.L. Phenotypic characterization of maize landraces from Sahel and Coastal West Africa reveals marked diversity and potential for genetic improvement. J. Crop. Improv. 2010, 34, 122-138. [CrossRef]

27. Hawtin, G.; Iwanaga, M.; Hodgkin, T. Genetic resources in breeding for adaptation. Euphytica 1996, 92, 255-266. [CrossRef]

28. Grobman, A.; Bonavia, D. Origin, Domestication, and Evolution of Maize. In Origin, Domestication, and Its Role in the Development Of Culture; Bonavia, D., Ed.; Cambridge University Press: Cambridge, UK, 2013; pp. 329-486. 
29. Katna, G.; Sood, V.K. Plant Genetic Resources, Traditional Knowledge and Their Use in Crop Improvement. In Plant Genetic Resources and Traditional Knowledge for Food Security; Salgotra, R.K., Gupta, B.B., Eds.; Springer Nature Singapore Private Limited: Singapore, 2015; pp. 23-38.

30. Yamasaki, M.; Wright, S.I.; McMullen, M.D. Genomic screening for artificial selection during domestication and improvement in maize. Ann. Bot. 2007, 100, 967-973. [CrossRef]

31. Taller, J.M.; Bernardo, R. Diverse adapted populations for improving northern maize inbreds. Crop Sci. 2004, 44, 1444-1449. [CrossRef]

32. Djemel, A.; Revilla, P.; Hanifi-Mekliche, L.; Malvar, R.A.; Alvarez, A.; Khelifi, L. Maize (Zea mays L.) from the Saharan oasis: Adaptation to temperate areas and agronomic performance. Genet. Resour. Crop Evol. 2012, 59, 1493-1504. [CrossRef]

33. Aci, M.M.; Revilla, P.; Morsli, A.; Djemel, A.; Belalia, N.; Kadri, Y.; Khelifi-Saloui, M.; Ordás, B.; Khelifi, L. Genetic diversity in Algerian maize (Zea mays L.) landraces using SSR markers. Maydica 2013, 58, 304-310.

34. Beyene, Y.; Botha, A.M.; Myburg, A.A. Genetic diversity among traditional ethiopian highland maize accessions assessed by simple sequence repeat (SSR) markers. Genet. Resour. Crop Evol. 2006, 53, 1579-1588. [CrossRef]

35. Djemel, A.; Cherchali, F.Z.; Benchikh-Le-Hocine, M.; Malvar, R.A.; Revilla, P. Assessment of drought tolerance among Algerian maize populations from oases of the Saharan. Euphytica 2018, 214, 149. [CrossRef]

36. Djemel, A.; Álvarez-Iglesias, L.; Santiago, R.; Malvar, R.A.; Pedrol, N.; Revilla, P. Algerian maize populations from the Sahara Desert as potential sources of drought tolerance. Acta Physiol. Plant. 2019, 41, 12. [CrossRef]

37. Maafi, O.; Revilla, P.; Álvarez-Iglesias, L.; Malvar, R.A.; Djemel, A. Adaptation assessment of drought tolerant maize populations from the Sahara in both shores of the Mediterranean Sea. Euphytica 2021. under review.

38. Aci, M.M.; Lupini, A.; Mauceri, A.; Morsli, A.; Khelifi, L.; Sunseri, F. Genetic variation and structure of maize populations from Saoura and Gourara oasis in Algerian Sahara. BMC Genet. 2018, 19, 1-10. [CrossRef] [PubMed]

39. Belalia, N.; Lupini, A.; Djemel, A.; Morsli, A.; Mauceri, A.; Lotti, C.; Khelifi-Slaoui, M.; Khelifi, L.; Sunseri, F. Analysis of genetic diversity and population structure in Saharan maize (Zea mays L.) populations using phenotypic traits and SSR markers. Genet. Resour. Crop. Evol. 2019, 66, 243-257. [CrossRef]

40. Cherchali, F.Z.; Ordás, B.; Revilla, P.; Pedrol, N.; Djemel, A. Heterotic Patterns among Algerian, US Corn Belt, and European Flint Maize Populations under the Mediterranean Conditions of North Africa. Crop Sci. 2018, 58, 2422-2432. [CrossRef]

41. SAS Institute. The SAS System for Windows; Release 9.4; SAS Institue: Cary, NC, USA, 2015.

42. Gardner, C.; Eberhart, S. Analysis and interpretation of the variety cross diallel and related populations. Biometrics 1966, 22, 439-452. [CrossRef]

43. Zhang, Y.; Kang, M.S.; Lamkey, K.R. DIALLEL-SAS05: A comprehensive program for Griffing's and Gardner-Eberhart analyses. Agron. J. 2005, 97, 1097-1106. [CrossRef]

44. Zeleke, K.; Demissew, A.; Wosene, G. Heterosis and combining ability of highland adapted maize (Zea mays L.) DH lines for desirable agronomic traits. Afr. J. Plant Sci. 2020, 14, 121-133. [CrossRef]

45. Badu-Apraku, B.; Akinwale, R.O.; Franco, J.; Oyekunle, M. Assessment of reliability of secondary traits in selecting for improved grain yield in drought and low-nitrogen environments. Crop Sci. 2012, 52, 2050-2062. [CrossRef]

46. Mogesse, W.; Zelleke, H.; Nigussie, M. General and specific combing ability of maize ( Zea mays L.) inbred line for grain yield and yield related traits using $8 \times 8$ diallel crosses. Am. J. Biosci. 2020, 8, 45-56. [CrossRef]

47. Chiuta, N.E.; Mutengwa, C.S. Combining ability of quality protein maize inbred lines for yield and morpho-agronomic traits under optimum as well as combined drought and heat-stressed conditions. Agronomy 2020, 10, 184. [CrossRef]

48. Oyekale, S.A.; Badu-Apraku, B.; Adetimirin, V.O. Combining ability of extra-early biofortified maize inbreds under Striga infestation and low soil nitrogen. Crop Sci. 2020, 60, 1925-1945. [CrossRef] [PubMed]

49. Yan, W.; Hunt, L.A. Genotype by Environment Interaction and Crop Yield. In Plant Breeding Reviews; Janick, J., Ed.; John Wiley \& Sons: Oxford, UK, 2010; pp. 135-178.

50. Noëlle, M.A.H.; Richard, K.; Vernon, G.; Martin, Y.A.; Laouali, M.N.; Liliane, T.N.; Godswill, N.-N. Combining Ability and Gene Action of Tropical Maize (Zea mays L.) Inbred Lines under Low and High Nitrogen Conditions. J. Agric. Sci. 2017, 9, 222-235. [CrossRef]

51. Ribeiro, P.; Apraku, B.B.; Gracen, V.; Danquah, E.; Afriyie-Debrah, C.; Obeng-Dankwa, K.; Toyinbo, J. Combining ability and testcross performance of low $\mathrm{N}$ tolerant intermediate maize inbred lines under low soil nitrogen and optimal environments. $J$. Agric. Sci. 2020, 158, 351-370. [CrossRef]

52. Amegbor, I.K.; Badu-Apraku, B.; Adu, G.B.; Adjebeng-Danquah, J.; Toyinbo, J. Combining ability of extra-early maize inbreds derived from a cross between maize and zea diploperennis and hybrid performance under contrasting environments. Agronomy 2020, 10, 1069. [CrossRef]

53. Alphonse, N.; Edema, R.; Asea, G.; Gibson, P. Combining Ability and Genotype By Environment interaction of selected maize inbred lines for performance under low nitrogen and drought stress. Afr. Crop Sci. Conf. Proc. 2011, 10, 1-4.

54. Umar, U.U.; Ado, S.G.; Aba, D.A.; Bugaje, S.M. Estimates of combining ability and gene action in maize (Zea mays L.) under water stress and non-stress conditions. J. Biol. Agric. Healthc. 2014, 4, 247-253. [CrossRef]

55. Njeri, S.G.; Makumbi, D.; Warburton, M.L.; Diallo, A.; Jumbo, M.B.; Chemining'Wa, G. Genetic analysis of tropical quality protein maize (Zea mays L.) germplasm. Euphytica 2017, 213, 261. [CrossRef] 
56. Meseka, S.K.; Menkir, A.; Ibrahim, A.E.S.; Ajala, S.O. Genetic analysis of performance of maize inbred lines selected for tolerance to drought under low nitrogen. Maydica 2006, 51, 487-495.

57. Beck, D.L.; Vasal, S.K.; Crossa, J. Heterosis and combining ability of CIMMYT's tropical early and intermediate maturity maize (Zea mays L.) germplasm. Maydica 1990, 35, 279-285.

58. Badu-Apraku, B.; Fakorede, M.; Talabi, A.; Oyekunle, M.; Aderounmu, M.; Lum, A.; Ribeiro, P.; Adu, G.; Toyinbo, J. Genetic studies of extra-early provitamin-A maize inbred lines and their hybrids in multiple environments. Crop. Sci. 2020, 60, 1325-1345. [CrossRef] [PubMed]

59. Makumbi, D. Phenotypic and Genotypic Characterization of White Maize Inbreds, Hybrids and Synthetics under Stress and Non-Stress Environments. Ph.D. Thesis, Texas A\&M University, College Station, TX, USA, August 2005.

60. Oyetunde, O.A.; Badu-Apraku, B.; Ariyo, O.J.; Alake, C.O. Efficiencies of heterotic grouping maturing maize inbred lines. Agronomy 2020, 10, 1198. [CrossRef] 